\title{
Diversity Management Strategies and Quality Decisions in the Banking Sector
}

\author{
Zachary Bolo Awino \\ Department of Business Administration, School of Business, University of Nairobi, Nairobi, Kenya
}

\begin{abstract}
The Top Management Team diversity discussed in the study is the extent to which the executive team is heterogeneous with respect to gender, age, academic qualifications, tenure, professional qualifications and functional backgrounds and how this aids quality decisions in the banking sector. Furthermore the study suggests that top management characteristics, specifically their demographic configuration could impair decision making which is the main objective and focus. Other researchers have shown that workforce diversity can provide positive organizational outcomes such as increased morale, higher satisfaction, greater commitment and improved performance. The questionnaire was the main tool of data collection. Cross sectional survey was adapted as the methodology of research where the target respondents were Heads of Human Resources in 43 banks. The data was analyzed using descriptive statistics and regression analysis. The TMT diversity attributes under study were age, gender, academic qualifications, tenure in the bank and at the top, professional and functional background diversity. These variables were used to determine the existing gaps in knowledge, whether these attributes were crucial in making quality decisions. The study also used secondary data obtained from the Banking sector to measure performance in terms of increase in customer deposits, increase in average assets and profit before tax. The study established that tenure in the banking sector has a major implication in the quality of decisions made within the Top Management Teams of organizations.
\end{abstract}

Keywords: Diversity, top management team, quality decisions, organizational performance

\section{Introduction}

As organizations increasingly operate in a multinational and multicultural context, understanding how diversity in the composition of organization group affects outcomes such as satisfaction, creativity, turnover and performance is of increasing importance (Johnston \& Packer, 1987). Top Management Team and their importance as potential determinants of organization performance continue to be a focus of strategic management researchers (Carson et al., 2004). Morison (1992) argued that organizations need to become more diverse with the respect to their employees' characteristics to match the increased diversity of backgrounds represented in their markets. The potential cognitive benefits of having a heterogeneous group stem from the argument on the impact of diversity on creativity (Hoffman \& Maier, 1961) and requisite variety.

Researchers have argued that in service industries like banks where competition is stiff and new players enter easily, there is a constant need to think strategically (Willie et al., 1997). According to Milliken and Martins (1996), diverse groups may make higher quality decisions. Organizational scholars (Cox et al., 1991) have illustrated that demographic characteristics of TMT have the potential to result in strategic decision making, greater creativity, more innovation and the ability to reach more and different types of customers, which is important in the banking sector. Proponents of diversity maintain that different opinions provided by diverse groups lead to quality decisions (Cox, 1993; McLeod et al., 1992) as diverse teams are able to produce a wider range of solutions and decision criteria for strategic decisions.

Other researches however indicate that demographic variation signals variation in underlying and invisible cognitive processes. As a result, the decision making process by the Top Management Team can be a source of strive as many decisions are decided by majority vote. This type of decision often leads to 'winners' and 'losers'. The significant potential for conflict within TMT diversity can thus slow down the decision making process resulting to inability to remain competitive. An organizational culture which involves the executives as well as employees may lead to commitment and a strong sense of ownership. The people feel that they have input into decisions that will affect their work and see a direct connection to the goals of the organization (Fey \& Denison, 2003).

Irrefutable measurable benefits can also be derived from properly implemented policies and strategies to promote diversity management (Jamrog, 2002). The most evident measurable benefits are improved bottom line, competitive advantage, 
superior business performance, employee satisfaction and loyalty, strengthened relationship with multicultural communities and attracting the best and the brightest candidates (Ashforth \& Mael, 1989). Barney and Wright (1998) noted that in order for human capital to contribute to sustainable competitive advantage, it must create value, remain hard to imitate and appear rare. The current study focuses on the relationship between Top Management Team diversity and organization performance in the Commercial Banks in Kenya. The relationship is moderated by diversity management strategies and involvement culture.

\section{Top Management Team and Quality Decisions}

Carson et al., (2004) defined diversity as any attribute that humans are likely to tell themselves that another person is different from them while Miller et al. (1998) defined the Top Management Team as all executives who report to the Chief Executive Officer or Chief Operating Officer. Researchers have categorized diversity in different ways. One distinction is between diversity on observable or readily detectable attributes such as age and gender and diversity in respect to less visible or underlying attributes, such as education, functional backgrounds, and tenure in the organization, personality and values (Cumming et al., 1993; Tsui et at., 1992).

The Top Management Team diversity discussed in the study is the extent to which the executive team is heterogeneous with respect to gender, age, academic qualifications, tenure, professional qualifications and functional back grounds. Simons et al. (1999) studied the same factors if TMT diversity with the exception of gender and professional qualifications. Diversity is an increasingly important factor in organizational life as organizations worldwide become more diverse in terms of gender, race, ethnicity, age and other characteristics (Shaw \& Barrett-Power, 1998).

Hamrick and Mason, (1984) argued that TMT diversity would be best able to manage turbulent complex environments since team member differences may stimulate debate about the appropriate strategy, allow them to generate greater range of strategic alternatives and collectively better evaluate the feasibility of such alternatives. Bantel and Jackson (1989) concluded that when solving complex, non routine problems, groups are more effective when they comprise individuals with diverse skills, knowledge, abilities and perspectives. Pfeffer, (1983) found out that increases in TMT diversity enhances the organizations ability to acquire critical resources. Top Management Team diversity however comes with some challenges as initial conflict arises as the teams set norms (Carson et al., 2004). In order for teams to be efficient and effective, they must be able to reach a consensus regarding group decisions (Coll et. al., 2001).

According to Gilmore (1998), decision making is an incremental, subsequent process which does not necessarily happen at one point in time. It is the most hypothesized intervening variable in discussions of executive diversity and organization performance (Fredrickson \& Mitchell, 1984). When making complex decisions, organizations must evaluate what is at stake in making the decision and also the parties involved. It involves planning and implementation. The planning stage involves diagnosis of what is wrong or needs to be changed, developed and refined- the initial problem identification. Obstacles arise at this stage from clashes in organizational structure, personalities and group dynamics. Analytical obstacles emerge from uncertainty, complexity, business cycles and variability in risk attitudes while content obstacles stem from information overload, lack of data, biases or the existence of too many or too few alternatives

Before undertaking any analysis, it is important to identify a broad set of alternative directions for a strategic decision. The alternatives should be creative, comprehensive, significantly different and compelling. It is also critical to identify clear decision criteria which ensure that values fit the corporate philosophy. The alternatives can be evaluated through individual idea generation and group brain storming. Alternatives can be evaluated through use of small group synthesis of key evaluation points, large group reviews and prioritization. When reaching the final decision, one to one previews of results or recommendations are used. Insight focus dialogue is also used (Gilmore, 1998).

The next level involves managing the actual output of decision making. This turns strategies and plans into action in order to achieve set objectives. Proponents of diversity hold that differences among group members give rise to varied ideas, perspectives, knowledge and skills that can improve the ability to solve problems and accomplish their work (Polzer et al., 2002). Complex tasks require problem solving, have a high degree of uncertainty and have a few set procedures, while routine tasks have a low level of variability and are repetitive (Polzer et al., 2002).

This stage involves harmonizing activities, coordinating efforts, supervising material and, responding to change quickly and having alternative plans. In addition, successful implementation involves the ability to be adaptable in order to respond to customers and competitors' activities in times of change (Gilmore, 1998). Persistence and perseverance are necessary for continuous improvement in order to resist erosion from competitive behavior and maintain consistency to avoid slipping standards. 


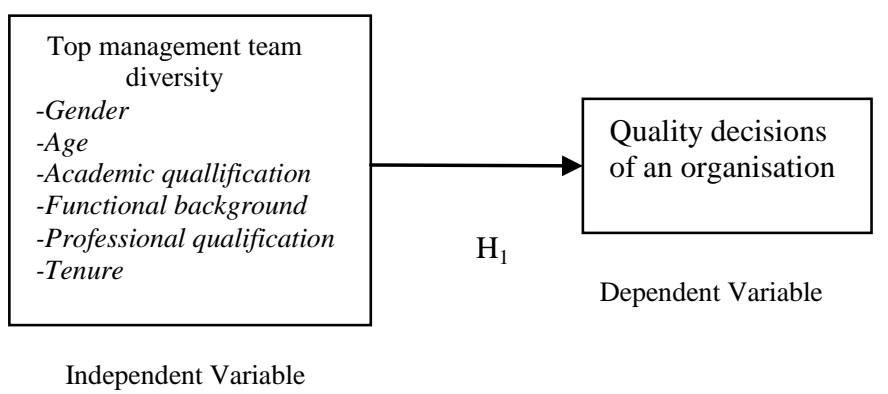

Figure 1. Conceptual model of top management diversity and quality decisions of an organisation

\section{Hypotheses}

$\boldsymbol{H}_{\boldsymbol{l}}$ : Top Management Teams diversity has an effect on quality decisions of an organisation.

\section{Methods and Results}

The study was a cross sectional survey which targeted all the 43 commercial banks in Kenya and offered the opportunity to collect data across the different banks at the same time. Cross sectional studies have been found to be robust in relationships studies (O'Sullivan \& Abela, 2007).

The primary data was collected using a semi structured questionnaire which was administered by research assistants face-to-face targeting heads of human resources in 43 banks in Kenya with 33 respondents returning the questionnaires, a response rate of $77 \%$. The questions asked were looking at the effect of Top Management Team Diversity on quality of decisions made by the managers. The questionnaire was divided into six parts, having both open and closed ended questions generated from previous empirical studies, theory and the researcher's own questions. The sections were divided into organizational profile, personal profile of the TMT and questions addressing the research objectives. The questionnaire was preferred because the respondents were literate and the same could be administered simultaneously to many respondents.

A five point likert type scale ranging from 5 to 1 , where 5 was "to a very great extent" and 1 referred to "not at all" was used to measure the performance of the banks. Pitts (2009) used a perceptual measure of performance which found a strong connection between subjective and objective measures of performance, with a correlation coefficient of .80 percent. The questionnaire was pre-tested in two of the commercial banks prior to the data collection to enable the researcher identify any unclear questions. It was realized that the questionnaire adequately measured what it was intended to measure with high degree of accuracy. Reliability and validity tests were carried on questionnaire. Reliability is a measure of the degree to which the study instruments yields consistent results or data after repeated trials and is influenced by random error. The reliability of the instrument was estimated using Cronbach's Alpha Coefficient which is used to assess the internal consistence or homogeneity among the research instrument items.

The alpha coefficient ranges in value from 0 to 1 and a high coefficient implies that the items correlate highly among themselves, that is, there is consistency among items in measuring the concept of interest (Mugenda \& Mugenda, 2003). Cronbach's Alpha Coefficient value of 0.7 is considered strong. In this case the value of 0.8 was established on the questionnaires

Validity is the accuracy and meaningfulness of inferences, which are based on the research results. It is the degree to which results obtained from the analysis of the data actually represent the phenomenon under study (Mugenda \& Mugenda, 2003). It is largely determined by the presence or absence of systematic error of data (non- random error). This study used face validity method where a panel of experts gave their input as to whether the instrument met the criterion.

Positivism approach seeks empirical regularities which are correlational between variables. The study between TMT diversity and quality decisions seeks to establish the relationship between these variables and also the strength of the relationships.

Further, a research design under the positivism approach should provide confidence to the scientific community that the findings derived from following the design capture the reality and possess high levels of reliability and validity (Kerlinger, 2007).

The banking sector was appropriate for the study due to the high level of competition and its impact on the economic growth of the other sectors through lending. There is also availability of data on the financial performance of banks in Kenya. The study targeted the Heads of Human Resources or equivalents who have insights on TMT diversity, diversity management strategies and organizational culture of their banks. The Head of Human Resources or equivalent was requested to provide the Bio data of the TMT which was available. Such 
an approach was used by Bantel and Jackson (1989) in their study on Top management and innovation in banking. Simple regression analysis was done to determine the effect of the factors which make up TMT diversity on the quality of decisions presented in Table 1.

Table 1. Effect of TMT diversity on quality of decisions

\begin{tabular}{llcl}
\hline \multicolumn{1}{c}{ Model } & \multicolumn{2}{c}{ Unstandardized Coefficients } & $\begin{array}{l}\text { Standardized } \\
\text { Coefficients }\end{array}$ \\
\hline (Constant) & $\mathrm{B}$ & Std. Error & Beta \\
Gender Diversity & 10.510 & 1.314 & \\
Age Diversity & -.438 & 2.059 & -.043 \\
Tenure diversity in bank service & -.383 & .792 & -.125 \\
Tenure diversity at top management & 1.231 & .558 & $\mathbf{. 5 6 1}$ \\
Academic diversity & -.778 & .735 & -.263 \\
Professional qualifications diversity & -.021 & .647 & -.007 \\
Functional backgrounds diversity & 1.037 & 2.062 & .123 \\
\hline Dependent Variable: QUALITY OF DECISIONS & .743 & 2.038 & .092 \\
$\mathrm{r}=474$ & & & \\
$\mathrm{R}$ Squared $=.225 \quad$ Durbin-Watson $=1.843$ & & & \\
$\mathrm{~F}=.911$ & & & \\
\hline${ }^{\mathrm{P}<.05}$ & & & \\
\hline
\end{tabular}

Table 1 presents the results of the regression analysis, with $\mathrm{r}=.474$ which shows a positive relationship between the factors making up TMT diversity and quality of decisions. The value of $\mathrm{R}^{2}$ is .225 , meaning that factors making up TMT diversity can explain 22.5 percent of quality of decisions. The rest, 77.5 percent is explained by other factors. The Durbin-Watson value is 1.843 , showing that the data is good for the analysis. The F ratio is .911 and is not significant meaning that the regression model does not predict the organization performance significantly well.

The results in Table 1 further show that tenure in the bank, has a significant effect on quality of decisions with $\beta=.561$ at $\mathrm{p}<.05$. Other effect which are positive though not significant on quality of decisions are professional qualifications diversity $(\beta=.123)$ and functional background $(\beta=.092)$. All the other factors of TMT diversity, namely, age, gender, academic qualifications, tenure at the top management have a negative effect on the quality of decisions, which was not significant. We can therefore conclude that Tenure at the bank has a significant effect on quality of decisions.

\section{Conclusion}

The study found out that tenure in the bank had a statistically significant effect on the quality of decisions made by a diverse TMT in the commercial banks in Kenya. The other factors making up TMT diversity did not have any significant effect on the quality of decisions.

Diversity among the TMT is widely assumed to influence the quality of decisions but empirical research on this linkage has been virtually limited. Past empirical research has shown that tenure can have both positive and negative effect on an organization. The common belief is that TMT members are naturally more comfortable with those they joined the banks with due to ease of identification or sense of belonging.

TMT with greater tenure are usually known to be more cohesive but less likely to change the status quo. Bantel and Jackson (1989) found a positive relationship between tenure and commitment to status quo. In a TMT composed of members who have worked for different periods, conflict is bound to happen as those who have worked for long feel that they have more institutional memory than those who have recently joined the team and as a result are committed to maintain things as they have always been. Status quo is likely to create a comfort zone, leaving no room for creativity and innovation which are critical components of the performance of banks.

The findings of this study show that TMT with diverse tenure has a significant effect on the quality of decisions. This means that diversity in tenure of the TMT is good for the performance of banks in Kenya. Banks should always ensure that they have a TMT which has worked for varying periods to draw the best. It is expected that those who have worked longer have valuable institutional memory while those who are newer bring in unique experiences in to the decision making process.

Generally, however, most of the factors which make up TMT do not have a significant effect on quality of decisions. This can be interpreted to mean that a diverse TMT may not be cohesive enough and thus do not involve people directly impacted by the decision nor make extensive use of eternal experts. It may further be argued that the TMT which is diverse doesn't consider all the possible causes of problems, either due to limited communication among members or lack of cohesiveness. This confirms the findings by 
O'Reilly et al. (1989). Hambrick's (1994) concept of behavioral integration suggests that TMT diversity affects cohesion in that individuals assume that those who agree with them also like them and vice versa.

Further, this study confirms that TMT diversity affects cohesion, which was described by O'Reilly et al. (1989) as the extent to which TMT like one another and stick up for each other. Members of teams which are not cohesive are more likely to challenge opinions by their colleagues. They further encourage debate and initiate investigations designed to uncover flaws in the reasoning of their colleagues (Miller et al., 1989). Team members are known to value group membership to the extent where fear of membership loss results to conformity and unquestioned acceptance of ideas which compromises the quality of decisions

Other empirical researchers have argued that when there are many disagreements about a threat or an opportunity, TMT are aware of more issues, more ways of viewing each issue and more alternative courses of action (Bantel \& Jackson, 1998). This results to in depth analyses and even hiring of experts to help solve the issues but if there is less disagreement, TMT is unlikely to consider the issues at depth. These arguments may result to reduced group think and more strategic options. Another argument that TMT diversity leads to quality of decisions states that disagreement in strategic decisions leads to greater willingness to expand resources necessary for more analyses, consultations and discussions (Miller et al., 1989). The findings of this study indicate otherwise therefore we can conclude that TMT diversity does not have a significant effect quality of decisions apart for tenure diversity in the bank which has a significant effect on quality of decisions.

\section{Implications of the study on Policy and Practice}

The study emphasizes the importance of how quality of decisions affects performance of banks positively. Policy and decision makers need to involve those who are impacted by decisions and also take time to determine the causes of major problems. In addition, the need to brain storm, generate many options, engage experts and make use of historical data add value in coming up with quality decisions.

While making the final decisions, TMT need to make contingency plans, compare several alternatives and promptly implement decisions in order to enhance the performance of the banks.

\section{References}

Adler, N. J. (1993). International dimensions of organizational behavior. Boston: PWS-KENT. American heritage dictionary of the English language. New York: HoughtonMifflin.

Bantel, K.A \& Jackson, S. E. (1989). Top management and innovations in Banking: Does the composition of top management make a difference?" Strategic Management Journal, 10:107-124.

Carson, M. C, Mosley, D. C \& Boyar, S.L. (2004). Performance gains through diverse top team management, Team Performance Management, 10: 21-126.

Coll Sambrhanya R. B \& Tucci L.A. (2001). Top management team composition, corporate ideology and organization performance. Mat Turter, 41(2), 109-129.

Cornelius, N. (1999). Human resource management: A managerial perspective, Thomson Business Press, Cornwall.

Cummings. A, Zhou. J \& Oldham, G.R. (1993). Demographic differences and employee work outcomes: Effects on multiple comparison. Paper presented at the annual meeting of the Academy of Management. Atlanta. GA.

Gilmore, A (1998). Quality in management decision making within a changing context. Journal of Management Development, 17(2), 106-120.

Hambrick, D.C. (1994). Top management groups: A conceptual integration and reconsideration of the team label.

Hambrick, D.C \& Mason, P. A. (1984). Upper echelons: The organization as a reflection of its top managers. Academy of management Review, 9: 193-206.

Kandola, R \& Fullerton, J. (1998). Diversity in action: Managing the Mosaic Wiltshire Cromwell Press.

Kerlinger, N. F. (2007). Foundations of behavior research, New Delhi: Surjeet Publications.

Kotter, J.P \& Heskett,J.L. (1992). Corporate culture and performance. Free Press, New York.

Miller, C.C., Linda M. B. \& William H.G (1998). Cognitive diversity among Upper-Echelon Executives: Implications for strategic decision processes, Academy of Management Journal, 19,39-58.

Morrison, A. (1992). The new leaders: Guidelines on leadership diversity in America. San Francisco: Jossey-Bass.

O'Reilly, C. A., Caldwell, D. F \& Barnett, W. P. (1989). Work group democracy, social integration, and turnover. Administrative Science Quarterly, 34: 21-37.

O’Sullivan, D \& Abela, V.A. (2007). Marketing performance measurement ability and organization performance. Journal of Marketing, 71: 79-93.

Pitts, D. (2009). Diversity management, job satisfaction and performance: Evidence from U.S federal agencies. Public Administration Review.

Pfeffer, J (1983). Organizational democracy. 3: 1-52 Greenwich CT JAI Press

Polzer, J. T., Laurie P. M \& William B.S (2002). Capitalizing on diversity: Interpersonal congruence in small work groups

Shaw, J. B \& Barrett-Power, E. (1998). The effects of diversity on small work group processes and Performance, Human Relations, 51 (10), 1307-1325.

Simons, T., Pelled, L.H \& K.A Smith. (1999). Making use of difference: Diversity, debate and decision comprehensiveness in Top Management Teams, Academy of Management Journal, 42(6), 662-673.

Tsui A. S, Egan T. D \& O'Reilly C. A (1992). Being different: Relational demography and organizational attachment. Administrative Science Quarterly, 37: 549- 579 